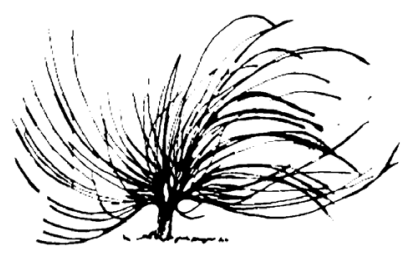

\title{
Propuesta de modelo estratégico que muestra los elementos esenciales de la innovación curricular
}

\author{
Rodrigo Urcid Puga ${ }^{1}$ \\ Tecnológico de Monterrey \\ México \\ rurcid@tec.mx
}

\begin{abstract}
Resumen
En el presente artículo, se hace una propuesta gráfica de los distintos elementos que deben considerarse al momento de pensar en la innovación curricular al interior de las universidades. Con este texto, se pretende mostrar el vínculo que existe entre algunas variantes educativas, sociales, culturales, económicas, etc. que se ven vinculadas con el tema al momento de crear nuevos enfoques educativos. El proyecto realizado se presenta mediante una metodología cualitativa con la herramienta documentada, se concibe como una investigación transversal en la que no se modificaron las variables. El resultado de este proyecto fue un modelo que comprueba que la innovación curricular no puede ser abordada desde un solo punto o tema, sino que incluye una serie de conceptos e interacción entre los mismos que no debe obviarse. Este análisis solo representa un acercamiento de los muchos existentes hacia este tópico; y que algunos de los estudios futuros pueden ser la comparación entre modelos tradicionales y los más innovadores, e incluso una serie de estudios cuantitativos que ayuden a entender la correlación y
\end{abstract}

Recibido: 27 de junio de 2019. Aprobado: 29 de abril de 2020.

http://dx.doi.org/10.15359/rep.15-2.11

1 Profesor de tiempo completo en el Departamento de Comunicación y Medios Digitales en la Escuela de Humanidades y Educación. Miembro del Sistema Nacional de Investigadores (SNI) Nivel "C". Dr. Planeación Estratégica y Dirección de Tecnologías por la Universidad Popular Autónoma del Estado de Puebla. ORCID: https://orcid.org/0000-0002-5102-3558 
relación existente entre profesorado y alumnado que opta por implementar y aceptar la innovación curricular al interior de las Instituciones de Educación Superior (IES).

Palabras clave: estudiantado, innovación curricular, profesorado, universidad

\begin{abstract}
In this article, a graphic proposal is made of the different elements that should be considered when thinking about curricular innovation within universities. This text aims to show the link that exists between some educational, social, cultural, economic variants, etc. that are linked in the subject when creating new educational approaches. The project carried out is presented through a qualitative methodology with the documented tool; it is conceived as a cross-sectional investigation in which the variables were not modified. The result of this project was a model that proves that curricular innovation cannot be approached from a single point or topic, but includes, series of concepts and interaction between them that should not be overlooked. This analysis only represents an approach of the many existing towards this topic and that some of the future studies may be the comparison between traditional and the most innovative ones, and even a series of quantitative studies that help to understand the correlation and / or existing relationship between teachers and students who choose to implement and accept curriculum innovation within the universities.
\end{abstract}

Keywords: Curricular innovation, students, teachers, university

\title{
Introducción
}

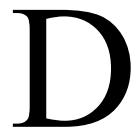
esde inicios del siglo XXI, la forma de entender al conocimiento ha cambiado de forma radical, y con ello se han puesto en marcha diversas modificaciones en el sector educativo, ya sea a través de las tecnologías, en los planes de estudio, en las aulas o en la forma de impartir cátedra. Al continuar en el espectro académico, la palabra innovación, se ha convertido en un elemento indispensable al momento de buscar revalorizar a la educación (Mateo y Vlachopoulos, 2013).

Así, la retórica de la innovación en las escuelas muestra argumentos acerca de la realidad en la actualidad, misma que puede ser 
incierta y compleja; lo anterior tiene como consecuencia, entre muchas otras, que los modelos educativos pierdan vigencia continuamente, y de ahí, se tenga la necesidad de revisar, de manera constante, la innovación escolar (Díaz-Barriga, 2012).

Por otro lado, la universidad responde a los distintos retos que plantea la sociedad y contexto en el cual se desenvuelve. Si bien las Instituciones de Educación Superior (IES) tienen objetivos como el preparar al estudiantado, fomentar y desarrollar las competencias en el alumnado, profesionalizar al profesorado, entre otros, no significa que no estén exentos de diversas dificultades; una de ellas, es el estancamiento en sus diversas áreas y la pérdida de actualidad que pueden tener; ante ello, lo ideal es crear unidades organizativas dedicadas a la innovación y a la mejora de la calidad de la enseñanza a través de diversas acciones y con la ayuda de múltiples herramientas (Adell, 2001).

\section{Problema de investigación}

Actualmente no se cuenta con un modelo o esquema que muestre de forma fehaciente los componentes de la innovación curricular; si bien, se encuentra material sustancial sobre el tópico en cuestión, no hay un gráfico que muestre la interacción de los diversos elementos que no solo llevan a la innovación curricular, sino la consecuencia positiva que esta puede tener, los riesgos que de ella emanan y cuáles son los stakeholders $^{2}$ o aspectos sociales que le caracterizan.

Sumado a lo anterior, la forma tan vertiginosa en la que el cambio educativo se presenta impide que la innovación curricular tenga un solo flujo, y por el contrario se tengan diversas vertientes desde las cuales puede ser entendida, esto trae como consecuencia que se escriba sobre el tema, pero queda un espacio para crear material que sirva como apoyo visual y ayude a esquematizar la importancia, repercusiones y características generales en esta materia.

Así, la relevancia de la temática que se expone radica en la importancia que la innovación tiene como dimensión inherente al sistema educativo; por ello, la idea de este modelo es resaltar la importancia de plantear la innovación curricular como un factor intrínseco y extrínseco tanto para el ámbito académico como para establecer premisas y condiciones para lograr los cambios necesarios.

2 También se les denomina grupos de interés. 


\section{Objetivo}

Proponer un modelo que muestre cómo puede desarrollarse y cuáles son los agentes que intervienen en la innovación curricular; entendiendo claro, que solo a través de la innovación las IES se puede alcanzar mejor calidad, ventaja competitiva y reconocimiento por parte de sus grupos de interés.

\section{Antecedentes}

El sistema educativo cambia como consecuencia de la propia sociedad, las modificaciones que deben de realizarse van en el propio sentido de lo que el sector educativo demanda; sin embargo, dichos cambios no deben ser forzados, por el contrario, estas mejoras deben ser constantes y no esperar a que la institución educativa tenga un notable rezago. Así, un ejemplo claro de ello es la innovación curricular, misma que se desprende de una serie de mejoras para que el estudiantado sea capaz de sacar el mejor provecho a su estadía en las universidades.

Este tema no es nuevo, se ha estudiado a fondo durante muchos años, $\mathrm{y}$ son diversos los autores que hacen aportaciones en cuanto a los beneficios de la misma; cabe denotar que si bien se tiene información al respecto, aún no se ha realizado un modelo en el que se proponga cómo es que la innovación curricular se ve entrelazada con una serie de elementos socioculturales y cómo es que, precisamente, no se ha dejado de lado las repercusiones que puede tener.

Así, un primer acercamiento es el que la UNESCO hace al término innovación considerándolo como "un proceso de destrucción creadora" (UNESCO, 2005, p. 62); sin embargo, esta definición carece de crítica, y limita la visión que pueda tener en referencia al cambio educativo a largo plazo. De ahí la necesidad de ampliar el concepto: la innovación necesita la creación de nuevas necesidades en la sociedad, esta, debe convencerse de las ventajas que puede obtener a partir de la misma, al tener como principal beneficio que el grado de conocimiento generado sea mayor (UNESCO, 2005).

Otra aproximación es la que Lugo (2008) propone, en la cual indica que la innovación está presente en los procesos de cambio en la mayoría de las universidades que suelen proponer ajustes en las estructuras, procesos y normativas; sobre todo, en la actitud de quienes la componen, así como por la forma en la crean vínculos con la sociedad. 
Durante el 2004, Zabalza, informaba que el aspecto crucial para lograr la innovación en una IES radicaba en la capacitación del profesorado, y que son varios los factores que influyen en el éxito de los diversos programas de formación académica, entre ellos está la mentalidad del profesorado, la disponibilidad de tiempo, existencia de referentes y ejemplos de buenas prácticas.

Desde otro punto de vista, al hacer un acercamiento, Conford (2001), busca conocer la adopción de la capacitación basada en competencias por las empresas australianas a partir de la innovación académica. En el año 2006, en Estados Unidos de América (EUA), Wright, Mcneill, Fry, Tan y Schempp, estudian la aplicación de innovación curricular y en la enseñanza en estudiantes de pedagogía en educación física. Para el 2009, Gregory y Morón realizan un estudio en Inglaterra enfocado en la adopción de tecnología e innovación para mejorar el aprendizaje en la educación superior. Durante el 2010, Borrego, Froyd y Hall, realizan un estudio que se enfoca en el conocimiento y adopción de innovaciones en la enseñanza de la ingeniería en los EUA.

En el contexto latinoamericano, Rosales (2012), afirma que la innovación se crea y tiene lugar bajo múltiples elementos, entre ellos: el salón de clases, o espacios que sirven de aula, el sistema administrativo, la comunidad en la que se desarrolla la institución educativa y, por supuesto, los grupos de interés que la conforman.

Finalmente, y en el contexto mexicano, Díaz-Barriga (2012), comenta que sin importar el nivel educativo, las instituciones educativas de este país priorizan la innovación educativa, pero, desafortunadamente la interpretación es radicalmente distinta, lo cual tiene como consecuencia que los proyectos encabezados por las universidades sean disonantes; la misma autora señala que algunos modelos considerados innovadores -flexibilización del currículo, educación por competencias, enseñanza basada en problemas y casos, la metodología de proyectos, tutorías académicas, etc.- deben ser contextualizados de acuerdo con los propios lineamientos de las instituciones educativas.

\section{Marco Teórico}

Para tener una investigación más robusta, se decide hacer un acercamiento profundo a la literatura que comprende diversos temas relacionados con la innovación curricular. En este sentido, y para 
tener una idea más clara de cómo se realiza este estudio, se presenta la tabla uno en la que se exponen los diversos tópicos que se trabajaron en esta investigación.

\section{Tabla 1.}

Temas abordados en la investigación

\begin{tabular}{|l|l|}
\hline \multicolumn{2}{|c|}{ OUTLINE TEÓRICO } \\
\hline Temas medulares & Temas complementarios \\
\hline Innovación curricular & Profesorado e innovación curricular \\
\hline Currículum & Evaluación por competencias \\
\hline Temas relacionados & Vinculación sociedad, universidad y mercado laboral \\
\hline Universidades & \\
\hline Universitarios & \\
\hline
\end{tabular}

Nota: Elaboración propia.

La innovación curricular está rodeada de una serie de temas que deben ser analizados para tener un mejor entendimiento de dicho concepto, por ello, y para lograr una base teórica más robusta, se presentan algunos tópicos que, de acuerdo con la literatura consultada se convierten en ejes fundamentales. Cabe notar que la investigación realizada se ajusta a ciertos conceptos e ideas como: innovación curricular, flexibilidad académica, educación superior, profesorado, currículum, y demás tópicos relacionados, además, se realiza una búsqueda limitada al periodo 2012-2019.

\section{Universidad}

Es imprescindible mencionar que las escuelas son instituciones socioculturales, y que la forma en que están organizadas y su legitimización "dependen cultural e históricamente de la visión de las sociedades sobre el propósito educativo" (Livingstone y Sefton, 2016, p. 30). Por ello, para entender que la escuela, es el elemento esencial de la educación formal, es necesario mencionar que el agente primario de esta es el alumnado (Gonnet, 2017).

En este sentido, una de las intenciones de las IES está tanto en la enseñanza de conocimientos, como en la creación de habilidades y aptitudes que satisfagan las necesidades del entorno laboral y de la vida cotidiana, el cual se ve influenciado por diversos factores sociales, locales, globales y mundiales (González y Miguel, 2017). 
Por otra parte, a la universidad se le puede dar el calificativo de espacio privilegiado de elaboración y experimentación curricular; esto porque como señala Ares (2015), es un espacio que determina que los cambios educativos sean el resultado de un proceso de deliberación y transacciones entre múltiples actores, tanto internos como externos, los cuales mantienen diversos opiniones y puntos de vista.

Así pues, la educación superior tiene múltiples responsabilidades, sobre todo si apela a la innovación educativa como valor diferenciador; algunas de estas son la responsabilidad social, la comprensión de cuestiones con diferentes aristas, involucrar dimensiones sociales, económicas, científicas y culturales, y la habilidad para responder a ellas (Castillo, Mendoza, Plasencia y Díaz, 2018). Así pues, las IES tienen una serie de características que las hacen parte medular de la sociedad en la que se desarrollan. Los grupos de interés que las comprenden van desde el personal administrativo hasta el profesorado y alumnado, padres de familia, etc. Por ello, para lograr que las universidades puedan profundizar en el tema de la innovación curricular es necesario que esta sea planeada y desarrollada con mesura para que tenga el impacto deseado.

Por otra parte, un tema que se desprende del modo en que las universidades se componen es la forma en la que el profesorado desarrolla y aplica diversas técnicas didácticas, esto, claro, si se entiende que uno de los elementos que parten de la innovación curricular es la metodología y el estilo en que imparte cátedra.

En este sentido, el profesor se convierte en un mediador, la tarea fundamental se centra en crear los entornos y experiencias de aprendizaje idóneos. Para tener un mejor resultado, se tienen diversos métodos, y por lo regular se utiliza una metodología innovadora, que permita que el alumnado adquiera los resultados de aprendizaje esperados y desarrollar un aprendizaje activo y comprometido.

Por ello, también existen otras técnicas didácticas que suelen promover la innovación no solo curricular, sino del aprendizaje, algunas de ellas son: el trabajo cooperativo, el aprendizaje basado en problemas y en proyectos, el estudio de casos, los trabajos de investigación, etc., todos, de la mano de una metodología expositiva de calidad (Zabalza, 2012), según se cita en Gargallo, Pérez, Verde y García (2017).

De la mano de la innovación curricular y de las técnicas didácticas, se encuentra el alineamiento constructivo, el cual, de acuerdo con Biggs (2005), sostiene que las distintas partes del proceso de enseñanza 
y aprendizaje trabajan de forma coordinada, de tal forma que tanto los métodos de enseñanza como los procedimientos de evaluación, se unen para lograr las competencias y resultados de aprendizaje previstos.

\section{Universitarios}

Como ya se ha mencionado, el principal grupo de interés que tienen las universidades es el estudiantado, sobre todo porque es en ellos en quienes se enfoca la innovación curricular; esta, se basa en las necesidades que puedan tener y en los procesos de enseñanza y aprendizaje que de esta se desprenden. Por ello, en los siguientes párrafos se plasman las principales características que posee, actualmente, el alumnado.

Primero que nada, se debe señalar que el alumnado cuenta con experiencias y conocimientos previos que inciden en los procesos de aprendizaje; estos suelen ser desarrollados en los diferentes niveles educativos (Ausubel, Novak, y Hanesian, 2013).

Del mismo modo, el aprendizaje del alumnado es significativo cuando los contenidos se relacionan de modo flexible y no bajo una secuencia. Lo anterior puede llevarse a cabo si este posee la adecuada estructura cognitiva, determinados conceptos e ideas correctamente formadas y definidas para lograr el aprendizaje deseado; todo ello, le sirve para tener una mejor interacción con la información.

Así pues, el aprendizaje significativo ocurre cuando la nueva información logra un vínculo con algún concepto que el estudiante tiene en su propia estructura cognitiva, a partir de esto, se tienen nuevas ideas, conceptos y proposiciones que se relacionan con otras ideas (Bicocca, 2017). Lo anterior se menciona para tener una idea más clara de cómo es el proceso de adquisición de conocimientos por parte del estudiantado, esto claro, al considerar que la innovación curricular basa mucho de su desarrollo en este proceso.

Por otra parte, y como característica inherente del estudiantado, este grupo suele manifestar cierta actitud para relacionar el nuevo conocimiento con su estructura cognoscitiva; es decir, son los educandos, quienes logran ciertas técnicas de estudio que influyen de manera directa en los procesos de apropiación del conocimiento (Ausubel, Novak, y Hanesian, 2013).

En cuanto a la gestión del aprendizaje, la disonancia entre lo que se aprende en clase y en la vida cotidiana no es algo nuevo, pero cada vez cobra mayor relevancia. Actualmente se tiene una postura muy 
escolarizada del aprendizaje que suele marginalizar los conocimientos que los jóvenes obtienen a través de los medios y plataformas digitales (Pereira, Fillol y Moura, 2019).

En este sentido, no se puede dejar de lado que los medios digitales acentúan el intercambio de información, que, aunque no siempre es el idóneo, dicho proceso está presente y de una u otra forma, facilita la creación y transmisión de conocimiento y participación, misma que se caracteriza por la afinidad en línea, creación y circulación de redes y contenidos, de y entre sus miembros (Jenkins, Purushotma, Weigel, Clinton, y Robinson, 2009).

Al retomar el tema de los hábitos de estudio, es necesario recordar que estos influyen en los resultados que el estudiantado tiene en el nivel escolar, especialmente en el ámbito universitario (Martínez y Torres, 2005). De hecho, para Monedero (2014), los hábitos de estudio influyen en los resultados académicos del estudiantado. Dentro de las más empleadas se encuentra: el subrayado, las notas al margen, el resumen, la síntesis, el esquema de contenido, las fichas y, por supuesto tomar apuntes (Bicocca, 2017).

\section{Profesorado e innovación educativa}

El siguiente grupo de interés inmediato para las IES es el profesorado; en este sentido, cobran una vital importancia pues son quienes llevan -a través de diversos canales-, el conocimiento al alumnado. De ahí la necesidad de dedicarle una serie de párrafos para describir los elementos primordiales que les caracterizan.

Según Elliot (2008), el profesorado facilita los medios para que el alumnado logre un desarrollo y así ejerza una ciudadanía activa; sin embargo, este debe tener en cuenta sus fortalezas y debilidades para poder realizar sus labores de forma idónea; mismas que se presentan a partir de la creación de metodologías, de tiempo para realizar actividades, uso de técnicas y recursos, entre otras cuestiones didácticas; finalmente, el profesorado se ocupa del flujo comunicativo para facilitar el trabajo dentro y fuera del aula.

Por otra parte, el profesorado debe estar consciente de que los programas que ofrecen, los materiales a disposición del alumnado, las evaluaciones, etc., todo ello, está disponible al alcance de los públicos interesados; lo anterior debe significar mayor transparencia y calidad en lo que se imparte (Zabalza, 2004). 
Del mismo modo, el profesorado universitario requiere un estilo de formación específico, mismo que no suele coincidir con otros niveles educativos, esto, porque quienes integran las IES poseen peculiaridades claramente definidas y sus tradiciones profesionales son específicas (Rosales, 2012).

Para finalizar el tema del profesorado, es imperante mencionar que este grupo de interés requiere actualización constante, la cual conlleva retos, como el cambio en la forma de impartir cátedra, potenciar el aprendizaje autónomo pero guiado de los estudiantes y el organizar el aprendizaje basado en competencias (De la Torre, Rico, Escolar, Palmero y Jiménez, 2016).

\section{Currículum}

El siguiente tema que compete a la innovación curricular es el propio término de lo que es un currículum, sobre todo para lograr una mejor comprensión de cómo puede presentarse y las consecuencias que dicho concepto representa; en este sentido, Gimeno (2017), informa que el currículum es un programa de actividades centralizadas, debidamente secuencializadas, ordenadas metodológicamente.

$\mathrm{Al}$ currículum, también se le conoce como una serie de tareas y destrezas que deben desarrollarse; en ocasiones se entiende como un programa que proporciona contenidos y valores para que el estudiantado mejore el contexto en el cual se desarrolla, y así se logre incrementar el nivel cultural de una sociedad (Santander y Hormozábal, 2015).

Así una vez señalado el concepto de currículum es imperante entender que su desarrollo y reforma se acrecienta con el propio cambio educativo de la sociedad; concretamente, la reforma curricular aparece como el primer paso en la modificación de las políticas educativas en cada etapa institucional (Díaz-Barriga, 2003).

Otra arista del currículum se relaciona con el propio conocimiento académico, lo cual afecta su configuración en las dinámicas de generación, aplicación y crítica del conocimiento científico. Lo anterior, prácticamente es un efecto esencial de la universidad de finales del siglo XX e inicios del XXI (Lucarelli, Nepomneschi, Abal, Donato, Finkelstein y Faranda, 2000). En la actualidad, la innovación curricular, ha transformado el enfoque académico; el ejemplo más claro es el enfoque por competencias aplicado a la elaboración de los planes de estudios (Collazo, 2010). 
Al considerar lo anterior, es necesario lograr un currículum que incluya lo necesario para que la sociedad tenga de una formación básica integral que fomente aprendizajes y competencias para que el ser humano se desenvuelva en la vida (Casanova, 2012). Así, el diseño curricular supone una fusión entre lo teórico y lo práctico que la escuela, en colaboración con su entorno, oferte al alumnado para integrarse en su contexto.

Finalmente, el tema académico se encuentra en una etapa en la que el currículum responde a las demandas de una educación internacional, el cambio de la sociedad, la dinámica de vida y las propias necesidades de los empleadores (Pinar, 2011).

\section{Innovación curricular}

Una vez expuestos todos los temas anteriores, se puede tocar el eje primario de esta investigación, la innovación curricular; hay que acotar que el concepto de innovación se puede entender como la introducción de cambios justificados (Zabalza, 2004). Ahora bien, en el nivel de docencia, la innovación sugiere la aplicación de tres elementos: apertura, actualización y mejora. La primera, está unida a la flexibilidad, la segunda tiene que ver con estar al día, y a la tercera, se le puede entender como un compromiso con el ámbito educativo.

Por otra parte, se deben rescatar dos condiciones básicas de la innovación universitaria; una de ellas es la practicidad, en este sentido, debe ser viable, y, práctica, es decir, con resultados plausibles. En definitiva, actualmente se está ante una innovación cuando se tiene un proceso de cambio adecuadamente fundamentado; todo en aras de la mejora y actualización de las actividades y dispositivos formativos (Santander y Hormozábal, 2015).

De manera más específica, la innovación curricular no es un tema reciente, se concibe como la sedimentación de una constante valoración de la docencia que ha logrado cambiar de forma positiva durante los últimos años (Díaz-Barriga, 2012). Este término, confronta a las IES y a los académicos en un proceso, en el cual la docencia se asume como una actividad grupal y colegiada, y que crea espacios para la transversalidad y la integración institucional (Santander y Hormazábal, 2015).

La innovación curricular le da mayor énfasis a la flexibilización de las carreras universitarias y al diseño de planes de estudios de acuerdo con el enfoque curricular por competencias. Lo anterior lleva a 
puntos de vista polarizados, connotaciones y derivaciones en cuanto a lo que es la educación (Díaz-Barriga, 2006).

En ocasiones, la innovación curricular va ligada a las novedades educativas, pero carece de una reflexión sobre sus implicaciones en las estructuras curriculares, e incluso a la realidad de los espacios educativos. Por ello, debe entenderse como la necesidad de un cambio en paradigmas y prácticas sociales y educativas, la cual es producto de reflexión y apropiación de las personas involucradas en ella (Díaz-Barriga y Lugo, 2003).

Un elemento fundamental de la innovación curricular debe ser dejar de lado el tradicional modelo de enfocarse en los libros de texto, y optar por otros recursos tecnológicos que ayuden tanto al profesorado como al estudiantado a establecer una relación más dinámica en la que se utilicen diversos recursos en el proceso de enseñanza y aprendizaje.

Así pues, la innovación curricular debe incluir medidas reales que permitan y faciliten el trabajo individual y colectivo. Hay que incorporar nuevas metodologías y recursos didácticos, replantearse el papel de las Tecnologías de Información y Comunicación -TIC- y establecer modelos de seguimiento académico que contribuyan a la regulación de los aprendizajes y al desarrollo emocional y social del alumnado (Mateo y Vlachopoulos, 2013).

Finalmente, la innovación curricular busca entender los procesos de reforma curricular como mecanismos complejos que implican un cambio sistémico en las distintas instituciones educativas; por ello, no hay que ignorar la importancia de las condiciones sociales y ambientales que favorecen el establecimiento de los hábitos de estudio del alumnado; lo anterior debe ser considerado en aras de entender y lograr la adecuada innovación curricular, y sobre todo, se debe considerar el ambiente que rodea el proceso de estudio, el cual puede ir desde el mobiliario, la temperatura, la iluminación, la ventilación, hasta la postura y planificación de dicho proceso (Díaz-Barriga, 2012).

\section{Evaluación por competencias}

Las competencias se convierten en un tema que tiene un acercamiento complejo, esto, debido a que son diversas las definiciones y usos que suelen tener, sumado a que al tener distintos grados de desarrollo al generalizarlas se torna complicado. En el nivel académico, aquellas que se encuentran inmersas en el currículum se constituyen en elementos 
básicos de este y logran un rol de referente curricular para la evaluación (Casanova, 2012).

Ahora bien, es imprescindible que al momento del diseño del currículum se considere la función de los objetivos que el alumnado debe alcanzar, pues es a través de estos que se comprueba si el estudiantado avanza o no en el dominio de las competencias (Gonnet, 2017).

De manera concreta, se puede afirmar que un currículo por competencias es aquel que, a partir de aquellas que demandan las empresas y organizaciones, se ocupa de fomentarlas en el alumnado; para ello, las IES deben centrar sus metodologías de enseñanza en la responsabilidad del aprendizaje del estudiantado, en donde el profesor, como ya se ha definido, se convierte en un guía (Santander y Hormozábal, 2015).

Si se utiliza un modelo educativo curricular basado en competencias, con diferentes modelos del aprendizaje como el conductismo, el funcionalismo, el constructivismo y el sistémico-complejo, es imperante lograr nuevos procesos de enseñanza, porque requieren cierta idoneidad para adaptarse a diferentes contextos que incluyan diversos conocimientos.

Así pues, una vez que se definen las competencias básicas que el estudiantado debe desarrollar en determinado periodo académico, se deben establecer los objetivos a los que tienen que llegar (Mateo y Vlachopoulos, 2013).

Para lograr dichos objetivos cada área o materia debe fijar sus objetivos generales; cada una de estas se estructura en programaciones y unidades didácticas, mismas que contienen objetivos específicos de logro que contribuyen coherentemente a la consecución de competencias; en aras de conseguir lo anterior, es preciso incorporar las competencias establecidas en el diseño de la titulación en el currículum y la forma en que impactan en su diseño (Lugo, 2008).

Se ha advertido que, de acuerdo con la manera en que son abordadas, su rol suele modificarse, y son diversas las que se han establecido de acuerdo con los fines académicos, sin embargo, con fines de vincularlas a la innovación curricular, algunas de las más esenciales -según diversos autores-, son: la competencia de titulación, experiencia labo$\mathrm{ral} /$ práctica (antes de egresar), conocimiento de idiomas extranjeros y habilidad para el aprendizaje y administración del tiempo, estas son especificadas a partir de las necesidades que tienen los empleadores (González y Miguel, 2017). 
Por otro lado, Martínez y González (2018), proponen una serie de competencias transversales que suelen ser citadas en diversas fuentes de información, estas pueden ser: trabajo en equipo, aprendizaje autónomo, resolución de problemas, conocimientos generales básicos y de la profesión, manejo de las TIC, compromiso ético, habilidades en las relaciones interpersonales, creatividad e innovación, gestión de la información y del tiempo, aplicar el conocimiento a la práctica, organización y planificación, liderazgo, capacidad de adaptación a nuevas situaciones, crítica y autocrítica, reconocimiento y respeto de la diversidad y multiculturalidad.

Los mismos autores señalan que también es necesario desarrollar en el estudiantado competencias como el conocimiento de una lengua extranjera, la iniciativa, el espíritu emprendedor, la toma de decisiones, las habilidades de investigación, el análisis y la síntesis, el trabajo en contextos internacionales, la preocupación por la calidad, la responsabilidad, el rendimiento bajo presión, la responsabilidad social y el compromiso ciudadano, la confianza en uno mismo, la motivación por el trabajo, la negociación la sensibilización hacia temas medioambientales, el conocimiento de otras culturas y costumbres de otros países, el diseño y la gestión de proyectos, la motivación de logro, el gestionar y conducir planes de vida, proyectos personales y profesionales y el afirmar derechos, intereses, límites, necesidades. En materia laboral, se busca que existan competencias relacionadas con la identificación, con la organización y sus valores, auto-comprensión y gestión y evaluación de riesgos (Martínez y González, 2018).

En síntesis, y para lograr una adecuada innovación curricular, todas las competencias -sin importar los fines- deben ser agrupadas por afinidad y complementariedad de significados; para este proceso, se deben tomar en cuenta una serie aspectos y matices que integran cada una de ellas según las denominaciones asignadas en los diversos planes de estudio, y por supuesto, deben estar sustentadas en un análisis bibliográfico exhaustivo del significado de cada una de ellas.

\section{Vinculo entre sociedad, universidad y el mercado laboral}

Finalmente, un tema que debe ser mencionado en este marco teórico es la forma en la cual la innovación curricular se relaciona con un determinado grupo social, los alcances que puede llegar a tener, las aristas que engloba y por supuesto, la forma en la que las 
IES crean un enlace entre estas y el contexto en el que se ha de desarrollar el alumnado.

Por otra parte, la relación entre la formación académica y vocacional, entre estudio y capacitación, es una constante en la educación superior; sin embargo, actualmente cobra mayor importancia debido a la simbiosis entre la economía-sociedad y la universidad (Neave, 2001). De esta forma se puede afirmar que la universidad no puede mantenerse al margen de la realidad laboral, por el contrario, debe ofrecer la inserción social y laboral del alumnado; lo anterior se ve reforzado y puede ser posible a través de una formación basada en competencias (Michavila, Martínez, Martín, García y Cruz, 2016).

\section{Metodología}

En esta sección se muestra el tipo de investigación realizada. Primero que nada, este análisis se llevó a cabo a través del análisis cualitativo. En este sentido, Cook y Reichardt (2016), informan que no existe una definición aceptada sobre este término. Este concepto se suele utilizar para referirse a:

1. La filosofía en general y a un enfoque de investigación

2. Una lógica de investigación

3. Conjunto específico de técnicas de investigación

En la mayor parte de los casos, los estudios basados en esta metodología utilizan pequeñas muestras, participantes o unidades que no son representativos de la población de la que fueron obtenidos. Al igual que la investigación cuantitativa, la cualitativa es una herramienta útil para la investigación de los medios masivos de comunicación cuando se reconocen sus limitaciones. Cabe notar que la investigación científica es un "estudio sistemático, controlado, empírico y crítico de las propuestas hipotéticas sobre las supuestas relaciones que existen entre los fenómenos observados" (Wimmer, y Dominick, 2011, p. 9).

Ahora bien, el tipo de estudio que se siguió fue descriptivo, el cual reseña las características o los rasgos de la situación o del fenómeno objeto de estudio; además se concibe como seccional o transversal, el cual se caracteriza por obtener información del objeto de estudio (población o muestra) una única vez en un momento dado (Hernández, Fernández y Baptista, 2014). 
Además, la investigación aquí presentada se concibe como un trabajo de corte documental, el cual está basado en ciertos conocimientos fundamentados, ya que en su mayoría son estudios o proyectos a realizar, con propuestas concretas y soluciones reales, no ficticias, aunque en ocasiones manejemos conocimientos empíricos, pero ya comprobados. De esta forma, la investigación social basada en documentos se dedica a reunir, seleccionar y analizar datos que están en forma de documentos producidos por la sociedad para estudiar un fenómeno determinado; también se conoce como investigación basada en fuentes secundarias (Gómez, Grau, y Ingellis, 2014).

Ahora bien, la selección y recopilación de información se presenta en dos vías primarias: a través de bases de datos electrónicas como EBSCO y Google Académico en donde se hicieron búsquedas de palabras clave como innovación, educación, flexibilidad curricular, innovación educativa, universidades, profesorado, estudiantado y demás temas que se abordan en esta investigación; cabe notar que se hizo una restricción al periodo que abarca del 2012 al 2018, aunque no se descartaron especialistas de años anteriores. También se trabajó con distinto material bibliográfico obtenido de librerías y bibliotecas.

Para la elaboración de este escrito se realiza un filtro por año y temática de acuerdo con las necesidades de este estudio; en este sentido, se analizaron más de cien referencias digitales y físicas, tanto en inglés como en español; cabe notar que este proyecto se desprende de una investigación doctoral que se realizó desde el 2013 al 2017.

\section{Resultados y discusión}

Como bien se ha explicado, el resultado primario al cual se llega es consecuencia del análisis teórico a las diferentes fuentes teóricas. Cabe notar, que, como se menciona en el apartado metodológico, a partir de una investigación documentada se plasman de manera gráfica los diversos elementos y conceptos que componen a la innovación curricular. Así, y para tener una mejor comprensión, se presenta la Figura 1 propuesta y después, la respectiva interpretación. 


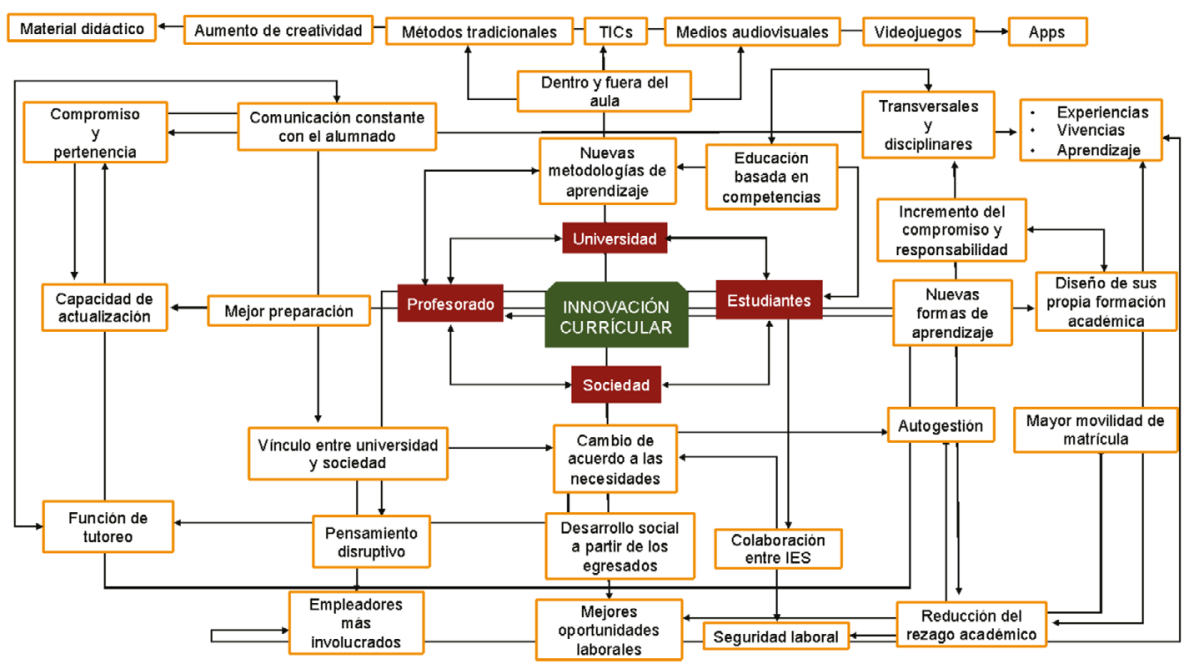

Figura 1. Elementos de la innovación curricular.

Nota: Elaboración propia.

La innovación curricular, de acuerdo con el análisis sostenido, presenta cuatro temas esenciales que muestran una cantidad de elementos que, de una u otra forma, interactúan de manera constante. De ahí que este modelo tenga una lectura multilineal, y no se caracterice por tener una jerarquía en particular; cabe notar que, si bien se presenta a la sociedad, al estudiantado, a la universidad y al profesorado como principales ejes de referencia, el gráfico puede iniciar o finalizar en cualesquiera de los conceptos o ideas que se muestran; es decir, se considera como un modelo flexible.

Sin embargo, y para fines de su comprensión, se cree prudente empezar a explicarlo desde la casilla que presenta uno de los principales grupos de interés los estudiantes. Esta idea se presenta a partir de que la innovación curricular debe ser planteada en aras de que el alumnado se vea beneficiado, en este sentido al incorporarla, este sector fomenta en sí mismo nuevas formas de aprendizaje, con lo que puede promover una autogestión y por ende diseñar su propia formación académica, en este sentido, se puede decir que hay un acompañamiento directo de las IES que le ayudan a incrementar su compromiso y responsabilidad.

El tener una autogestión del aprendizaje conlleva a que el rezago académico sea nulo, pues es el propio estudiantado quien busca 
avanzar en sus programas de estudio, los cuales él mismo ha diseñado gracias a la flexibilidad que la innovación curricular permite. Sumado a lo anterior, vale la pena mencionar que al reducir el aletargamiento del alumnado las universidades también tendrán un mayor movimiento de matrícula, con lo que el proceso de rotación estudiantil será continuo, y esto significa mejores beneficios económicos para las instituciones.

La siguiente casilla que se desprende de la idea central -innovación curricular-, es la de las IES; las instituciones académicas deben proceder a la creación de nuevas metodologías de aprendizaje, las cuales pueden ser tanto dentro como fuera del aula, esto no solo enriquece la experiencia del estudiantado en su paso por la etapa universitaria, sino que enriquece la manera en la que el profesorado se involucra en el proceso de enseñanza.

Así, este debe valerse tanto de elementos tradicionales como del uso de la tecnología; en este sentido, puede apoyarse en medios audiovisuales, videojuegos o aplicaciones; mientras que aumenta su creatividad y opta por diversos materiales didácticos. Al utilizar estos dos elementos primarios de enseñanza, se potencializa la educación basada en competencias, aspecto que, como ya se ha señalado es uno de los pilares de la innovación curricular.

Ahora bien, las competencias suelen ser divididas en transversales y disciplinares, mismas que, para que puedan tener un mejor entendimiento, deben ser agrupadas en aquellas que fomentan el aprendizaje, promueven las experiencias y fortalecen las vivencias académicas y prácticas; todas ellas, son apreciadas por la sociedad y por los empleadores.

Así, se puede llegar a la siguiente idea secundaria del modelo creado: sociedad; pero en este caso, se tiene una lectura inversa que parte, precisamente, del punto anterior; como consecuencia de una educación basada en competencias, se pueden tener mejores oportunidades laborales; mismas que detonan en trabajadores más involucrados con la empresa y fomenta una sociedad que colaboras con las IES.

Con lo anterior, la sociedad tiene un cambio de acuerdo con las necesidades actuales y contextuales a partir del desarrollo social y económico de los egresados, los cuales deben tener un pensamiento disruptivo, ya que los ciudadanos, no avanzan sino se tiene un sentido de evolución, el cual, se ve beneficiado y propiciado a partir del vínculo existente entre las universidades y la propia sociedad. 
Esta relación intrínseca que debe establecerse tiene que ser fomentada por el profesorado, el cual se convierte en la cuarta idea secundaria que se tiene en el modelo realizado; son los catedráticos, que a partir de la innovación curricular deben estar mejor preparados, y con una amplia capacidad de actualización. A partir de lo que se establece como un nuevo estilo de enseñanza, es que los catedráticos deben tener una función de mentores y evolucionar al proceso de acompañamiento, esto, conlleva a que se tenga un sentido de compromiso y pertenencia por parte de ellos hacia las IES en las cuales colaboran.

Este tutoreo va de la mano de una constante comunicación con el alumnado, aspecto que lleva a una interacción en el proceso de enseñanza y aprendizaje. Lo anterior corrobora lo que en un principio se señala, que la innovación curricular es un proceso que involucra la interacción de cuatro elementos esenciales que se ven en constante relación.

De esta forma, y una vez señalados los principales puntos por los cuales se conforma este modelo, es posible observar que prácticamente todas las casillas o ideas presentadas tienen una relación que no puede ser separada la una de la otra, pues para la innovación curricular es indispensable que la unión entre todos estos conceptos sea inherente.

Es importante entender que no se puede concebir a las IES sin el estudiantado, los cuales no se apartan de la sociedad, la cual depende de un profesorado correctamente capaz y con amplio criterio; de hecho, no es posible que la innovación curricular se entienda sin la correlación que tiene con el compromiso y responsabilidad del estudiantado, el cual tiene que estar en contacto con un profesorado que tenga la capacidad de mantener relaciones cercanas con los empleadores, los cuales deben promover la evolución de las sociedades.

\section{Conclusiones}

La idea de llevar a cabo este proyecto parte de establecer de manera visual cómo la innovación curricular está rodeada por una serie de factores sociales, económicos, culturales, educativos, gubernamentales, que, de una u otra forma permean al momento de plantearla al interior de las IES. Así, y de acuerdo con el objetivo planteado, se comprobó que es posible crear un modelo gráfico que muestre las distintas vertientes que se pueden desprender a partir de la innovación curricular.

La relación y conexión que se presenta en este modelo muestra que la innovación curricular debe ser abordada desde tantos aspectos 
sociales, económicos y académicos como sea posible, la interacción mostrada entre las casillas propuestas lo confirma y la mejor manera de entenderlo es mediante un concepto que debe ser el pilar de todo este cambio: la flexibilidad.

Como se puede ver, la innovación curricular impacta a diferentes aspectos que van desde lo social hasta lo económico, por ello, no debe obviarse y las IES deben tenerla presente en todo momento, sobre todo, si lo que se busca es que no solo la educación mantenga niveles de calidad y el proceso de enseñanza y aprendizaje sea mejor, sino que el país, la región y el contexto ciudadano se vea beneficiado y avance hacia un camino de prosperidad.

\section{Referencias}

Adell, J. (2001). El fomento de las nuevas tecnologías aplicadas a la formación presencial y a distancia en la universidad. Congreso Aplicación de las Nuevas Tecnologías en la Docencia Presencial y E-learning. Universitat de Valencia: Valencia.

Ares, J. (2015). Universidad: ¿Anarquía organizada? Montevideo: Librería de la Facultad de Humanidades y Ciencias de la Educación.

Ausubel, D. P., Novak, J. D. y Hanesian, H. (2013). Psicología Educativa: Un punto de vista cognoscitivo. Distrito Federal: Rinehart and Winston.

Bicocca, R.M. (2017). Análisis crítico-filosófico de las potencialidades educativas de la enseñanza basada en competencias. Educación y Educadores, 20(2), 267-281.

Biggs, J. (2005). Calidad del aprendizaje universitario. Madrid: Narcea. Borrego, M., Froyd, J. y Hall, S. (2010). Diffusion of engineering education innovations: a survey of awareness and adoption rates in us engineering departments. Journal of Engineering Education, 99(3), 185-207.

Casanova, M. A. (2012). El diseño curricular como factor de calidad educativa. Revista Iberoamericana sobre Calidad, Eficacia y Cambio en Educación, 0(4), 1-20.

Castillo, Y., Mendoza, B., Plasencia, E. y Díaz, C. (2018). Grado de responsabilidad social en estudiantes de una universidad pública. Revista Cubana de Educación Médica Superior, 32(2), 1-13.

Collazo, M. (2010). El currículo universitario como escenario de tensiones sociales y académicas. Didáskomai, 1, 5-23. 
Conford, I. (2001). Adoption of competency-based, training by business and industry, Knowledge Demands for the New Economy. Sidney: Australian Academic Press.

Cook, T. y Reichardt, Ch. (2016). Métodos cualitativos y cuantitativos en investigación evaluativa. Madrid: Ediciones Morata.

De la Torre, C., Rico, T., Escolar, C. Palmero, C. y Jiménez, A. (2016). La figura del profesor como agente de cambio en la configuración de la competencia emprendedora. Revista Interuniversitaria de Formación del Profesorado, 30(2), 131-144.

Díaz-Barriga, F. (2003). Currículum. Tensiones conceptuales y prácticas. Revista electrónica de investigación educativa, 5(2), 1-13.

Díaz-Barriga, F. (2006). El enfoque de competencias en la educación. ¿Una alternativa un disfraz de cambio? Perfiles Educativos, 28(111), 7-36.

Díaz-Barriga, F. (2012). Reformas curriculares y cambio sistémico: una articulación ausente pero necesaria para la innovación. Revista Iberoamericana de Educación Superior, II(7), 23-40.

Díaz-Barriga, F. y Lugo, E. (2003). La investigación curricular en México. La década de los noventa. México: Consejo Mexicano de Investigación Educativa.

Elliot, A. (2008). Handbook of approach and avoidance motivation. Nueva York: Taylor \& Francis.

Gargallo, B., Pérez, C. Verde, I. y García, E. (2017). Estilos de aprendizaje en estudiantes universitarios y enseñanza centrada en el aprendizaje. RELIEVE, 23(2), 1-22.

Gimeno, J. (2017). El currículum: una reflexión sobre la práctica. Madrid: Morata.

Gonnet, J. (2017). Educaçao para os media: As controvérsias fecundas. Porto: Porto Editora.

González, E. y Miguel, M. S. (2017). Egresados universitarios y su pertinencia para el ingreso a la empleabilidad empresarial o la autoempleabilidad. Revista Electrónica de Psicología Iztacala, $0(1), 168-185$.

Gregory, K. y Morón, S. (2009). Electronic assignment submission, student behaviour and experience. Engineering Education, 4(1), $16-28$.

Gutiérrez, J. (2008). Estrategias de autoaprendizaje. México: Editorial Trillas. 
Hernández, R., Fernández, C. y Baptista, P. (2014). Fundamentos de metodología de investigación. México: McGraw-Hill.

Jenkins, H., Purushotma, R., Weigel, M., Clinton, K. y Robinson, A. J. (2009). Confronting the challenges of participatory culture: media education for the $21^{\text {st }}$. Century. Cambridge: The MIT Press.

Livingstone, S. y Sefton, J. (2016). The class: living and learning in the digital age. New York: New York Unicersity Press.

Lucarelli, E., Nepomneschi, M., Abal, I., Donato, M., Finkelstein, C. y Faranda, C. (2000). El asesor pedagógico en la universidad. De la teoría pedagógica a la práctica en la formación. Buenos Aires: Paidós.

Lugo, E. (2008). Reformas universitarias: su impacto en la innovación curricular y la práctica docente. Distrito Federal: UAEM/ANUIES.

Maddox, H. (2010). Cómo estudiar. Barcelona: Editorial Oikos-tau.

Martínez, P. y González, N. (2018). Las competencias transversales en la universidad: propiedades psicométricas de un cuestionario. Educación XX1, 21(1), 231-262.

Martínez, V. y Torres, L. (2005). Análisis de los hábitos de estudio en una muestra de alumnos universitarios. Revista Iberoamericana de Educación, 36(7), 1-9.

Mateo, J. y Vlachopoulos, D. (2013). Reflexiones en torno al aprendizaje y a la evaluación en la universidad en el contexto de un nuevo paradigma para la educación superior. Educación XX1, 16(2), 183-208.

Michavila, F., Martínez, J.M., Martín, M., García, F.J. y Cruz, J. (2016). Barómetro de empleabilidad y empleo de los universitarios en España, 2015 (Primer informe de resultados). Madrid: Observatorio de Empleabilidad y Empleo Universitario.

Monedero, C. (2014). Dificultades de aprendizaje escolar. Madrid: Pirámide.

Neave, G. (2001). Educación Superior: historia y política. Barcelona: Gedisa.

Pereira, S., Fillol, J. y Moura, P. (2019). El aprendizaje de los jóvenes con medios digitales fuera de la escuela: de lo informal a lo formal. Comunicar. Revista Científica de Comunicación, 27(58), 41-50.

Pinar, W. (2011). Curriculum studies in Mexico. Nueva York: Palgrave-McMillan. 
Rosales, C. (2012). Contextos de la innovación educativa. Innovación educativa, 22, 9-21.

Santander, I. y Hormazábal, P. (2015). Difusión de la innovación curricular por competencias. UCMaule-Revista Académica, 8, 29-53.

UNESCO. (2005). Hacia las sociedades del conocimiento. Recuperado de http://www.lacult.unesco.org/docc/2005_hacia_las_soc_conocimiento.pdf

Wimmer, R. y Dominick, J. (2011). Introducción a la investigación de los medios masivos de la comunicación. México: Thomson Editores.

Wright, S., Mcneill, M., Fry, J., Tan, S. y Schempp, P. (2006). Implications of student teachers' implementation of a curricular innovation. Journal of Teaching in Physical Education, 5, 310-328.

Zabalza, M. (2004). Innovación en la enseñanza universitaria. Contextos educativos, 6(7), 113-136.

Zabalza, M. A. (2012). Metodología docente. REDU Revista de Docencia Universitaria, 9(3), 75-98. 
